\title{
New results for exotic quarkonia and heavy flavour with ATLAS
}

Ina Carli, on behalf of the ATLAS Collaboration*

Charles University, Faculty of Mathematics and Physics, IPNP, V Holesovickach 2, 18000 Prague, Czech Republic

\begin{abstract}
The measurements of production and properties of heavy flavoured hadrons can be used to probe our understanding of quantum chromodynamics and to search for physics beyond the Standard Model.

This paper summarises three recent results from the ATLAS experiment at the Large Hadron Collider. It includes search for an exotic state $X(5568)$ in the $B_{s}^{0} \pi^{ \pm}$final state, measurement of quarkonia production in $p \mathrm{~Pb}$ and $p p$ collisions and angular analysis of the rare decay $B^{0} \rightarrow K^{*} \mu^{+} \mu^{-}$.
\end{abstract}

Keywords: ATLAS, exotic quarkonia, quarkonia production, rare decays, $\mathrm{K}^{*}$ mumu

\section{Introduction}

The ATLAS experiment [1] at the Large Hadron Collider performs a wide range of precision measurements of the Standard Model (SM) processes and parameters and searches for hints of physics beyond the SM. The heavy-flavour program covers studies of production, spectroscopy and decay properties of $b$-hadrons and quarkonia, mostly focused on final states with muons.

The ATLAS detector is a general-purpose detector with a cylindrical geometry and nearly $4 \pi$ coverage in solid angle. The Inner Detector (ID) detects tracks of charged particles and consists of pixel, silicon strip and transition radiation subdetectors embedded in a $2 \mathrm{~T}$ solenoidal magnetic field. The ID is surrounded by a liquid-argon sampling electromagnetic calorimeter and a steel/scintillator tile hadronic calorimeter. The outermost Muon Spectrometer (MS) consists of three large toroids and a system of gaseous detectors for triggering and precise measurement of muon momenta.

Results presented here are based on dataset collected in 2011 (at centre-of-mass energy $\sqrt{s}=7 \mathrm{TeV}$ ) and

\footnotetext{
Copyright 2018 CERN for the benefit of the ATLAS Collaboration. CC-BY-4.0 license.

${ }^{*}$ Email address: Ina.Carli@cern.ch
}

2012 (at $\sqrt{s}=8 \mathrm{TeV}$ ) corresponding to a total integrated luminosity of $25 \mathrm{fb}^{-1}$. The $p P b$ collisions at $5.02 \mathrm{TeV}$ per nucleon pair were collected in $2013\left(L=28 \mathrm{nb}^{-1}\right)$ and the $p p$ collisions at the same energy in 2015 ( $L=$ $\left.25 \mathrm{pb}^{-1}\right)$.

\section{Search for a structure in the $B_{s}^{0} \pi^{ \pm}$invariant mass spectrum - tetraquark candidate $X(\mathbf{5 5 6 8})$}

Observation of a narrow structure $X(5568)$ was reported in 2016 by the D0 collaboration [2]. It was interpreted as a tetraquark composed of bsud quarks but searches by the LHCb, CMS and CDF collaborations show no signal $[3,4,5]$. The presented analysis by ATLAS [6] searches for X(5568) in a decay $X(5568) \rightarrow B_{s}^{0} \pi^{ \pm}, B_{s}^{0} \rightarrow J / \psi \phi$ in a dataset from $p p$ collisions collected in 2011 and 2012.

The $B_{s}^{0}$ candidates were reconstructed from two oppositely-charged muons fitted to a common vertex to form $J / \psi$ and two tracks in the ID from $\phi$-meson decay. Selection of $B_{s}^{O}$ candidates was done by applying cuts on proper decay time to suppress background and a cut on $p_{\mathrm{T}}$ which was used by D0. A track with pion mass hypothesis coming from the $B_{s}^{0}$ primary vertex was then added to form the $X$ candidate. 


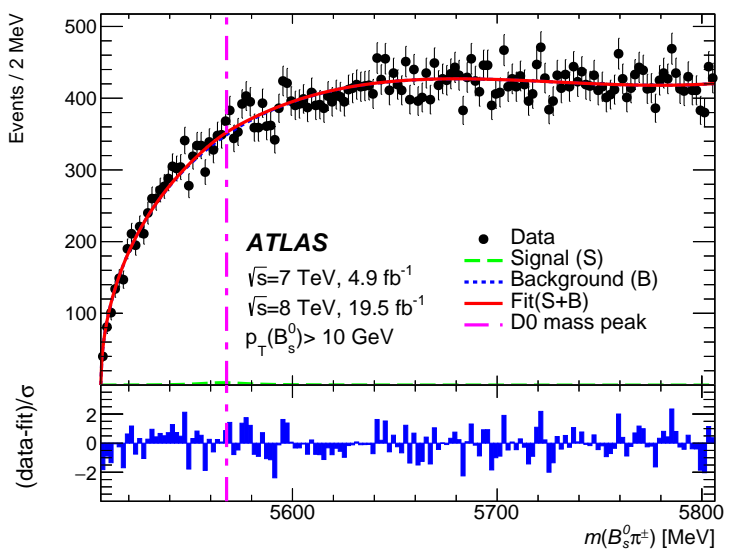

Figure 1: Fit of the $B_{s}^{0} \pi^{ \pm}$mass distribution for candidates with $p_{\mathrm{T}}\left(B_{s}^{0}\right)>10 \mathrm{GeV}$ : the probability density function (PDF) of background is defined as a threshold function and the $X$ signal is modelled by a Breit-Wigner function convoluted with Gaussian [6].

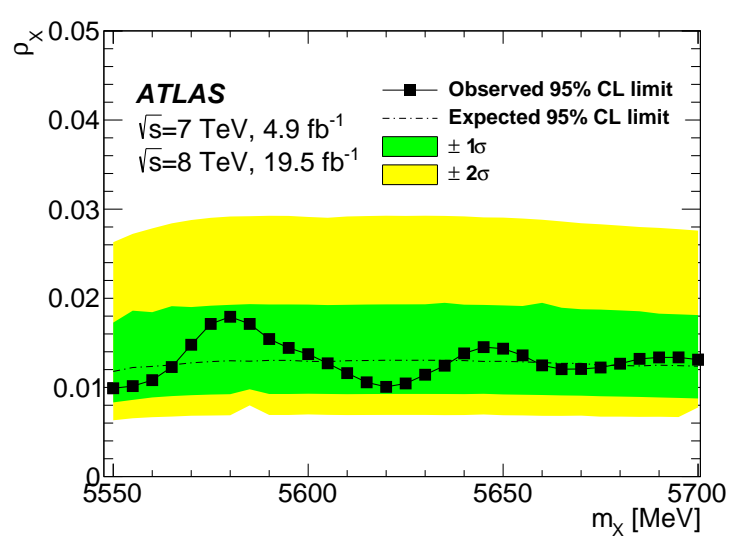

Figure 2: Upper limits on $\rho_{X}$ at $95 \% \mathrm{CL}$ for a narrow resonance decaying to $B_{s}^{0} \pi^{ \pm}$as reported by D0 in [2]. Figure is from Ref. [6].

Figure 1 shows the mass distribution of $B_{s}^{0} \pi^{ \pm}$candidates. No significant signal was found and the yields extracted from fit were used to set upper limits on the number of signal events and on the $X$ production rate relative to $B_{s}^{0} \rho_{X}$, assuming $X$ properties as reported by D0. The results are $N(X)<382$ and $\rho_{X}<0.0 .15$ for candidates with $p_{\mathrm{T}}\left(B_{s}^{0}\right)>10 \mathrm{GeV}$, and $N(X)<356$ and $\rho_{X}<0.0 .15$ for $p_{\mathrm{T}}\left(B_{s}^{0}\right)>15 \mathrm{GeV}$ at $95 \%$ confidence level.

A hypothesis test for the presence of a narrow $B_{s}^{0} \pi^{ \pm}$ peak was also performed for the mass range 5550$5700 \mathrm{MeV}$. The results are shown in Figure 2. The upper limits set on $\rho_{X}$ vary between 0.010 and 0.018 and are within $1 \sigma$ from the expected limit.

\section{Quarkonia production in $p \mathrm{~Pb}$ and $p p$ collisions}

Quarkonia are valuable probes of the properties of quark-gluon plasma (QGP) produced in heavy ion collisions. However, it is important to disentangle effects due to interaction between quarkonia and the deconfined and hot QGP from those coming from passage through cold nuclear matter (CNM). In proton-nucleus collisions, the formation of large regions of QGP is not expected and changes in the quarkonium production are attributed to CNM effects, such as modification of parton distribution functions, parton saturation and interaction with nuclear medium or absorption of the formed quark-antiquark pair in the nuclear medium.

ATLAS measured the production of $J / \psi, \psi(2 S)$ and $\Upsilon(n S)(n=1,2,3)$ in $p p$ and $p \mathrm{~Pb}$ collisions at centre-ofmass energy per nucleon pair of $5.02 \mathrm{TeV}$ [7]. Quarkonia were reconstructed in the dimuon final state and an unbinned maximum-likelihood fit of the mass distribution of selected candidates was used to extract the yields of bottomonia. To separate the prompt and nonprompt (from $b$-quark decays) components for $J / \psi$ and $\psi(2 S)$, the fit was performed simultaneously to mass and pseudo-proper lifetime distributions.

The obtained yields were used to measure the differential cross sections of quarkonia production in bins of $p_{\mathrm{T}}$ and rapidity, the nuclear modification factors $R_{p \mathrm{~Pb}}=$ $1 / 208 \cdot \sigma_{p \mathrm{~Pb}} / \sigma_{p p}$ and double ratios

$$
\rho_{p \mathrm{~Pb}}^{n S / 1 S}=\frac{R_{p \mathrm{~Pb}}(n S)}{R_{p \mathrm{~Pb}}(1 S)}=\frac{\sigma_{p \mathrm{~Pb}}(n S)}{\sigma_{p \mathrm{~Pb}}(1 S)} / \frac{\sigma_{p p}(n S)}{\sigma_{p p}(1 S)}
$$

and their dependence on quarkonium $p_{\mathrm{T}}$, protonnucleon centre-of-mass rapidity and collision centrality.

The nuclear modification factor for $\Upsilon(1 S)$ and prompt $J / \psi$ as a function of $p_{\mathrm{T}}$ is shown in Figure 3. The $R_{p \mathrm{~Pb}}$ is consistent with unity for $J / \psi$ but for $\Upsilon(I S)$ there is a suppression at low $p_{\mathrm{T}}$. This points to the modification of nuclear parton distribution functions.

Figure 4 shows the double ratios $\rho_{p \mathrm{~Pb}}^{n S / 1 S}$ as functions of the $p \mathrm{~Pb}$ collision centrality. A suppression of higher states is observed with a significance of $1 \sigma$ for $\psi(2 S)$ and at $2 \sigma$ for $\Upsilon(2 S)$ and $\Upsilon(3 S)$. The effect increases with centrality and suggests stronger CNM effects for excited quarkonia.

\section{Angular analysis of the decay $B^{0} \rightarrow K^{*} \mu^{+} \mu^{-}$}

The rare decay $B^{0} \rightarrow K^{*}\left(K^{+} \pi^{-}\right) \mu^{+} \mu^{-}$is mediated by flavour-changing neutral currents and in the SM it can occur only at the loop level. It is therefore sensitive to contributions from physics beyond the SM. 


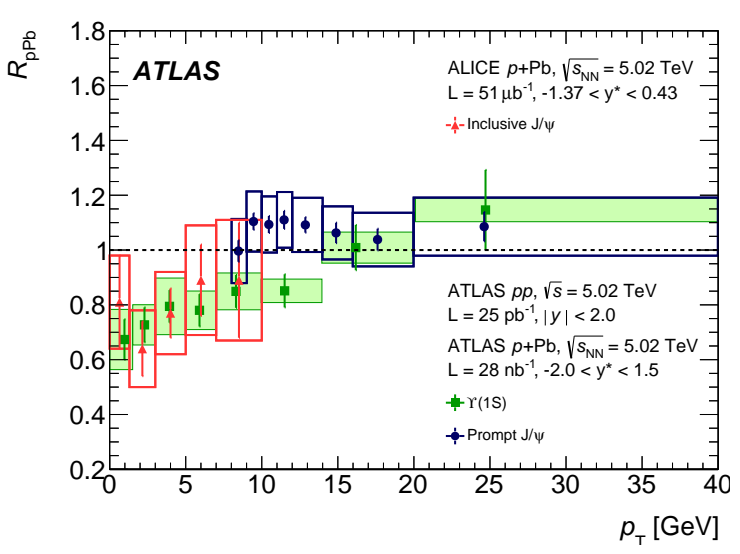

Figure 3: Nuclear modification factor as a function of $p_{\mathrm{T}}$ for $\Upsilon(1 S)$ and prompt $J / \psi$ [7], together with results from the ALICE experiment [8]. Vertical error bars cover the statistical uncertainties and horizontal error bars represent the bin size and vertical and horizontal size of boxes represent the systematic uncertainties and bin sizes, respectively.
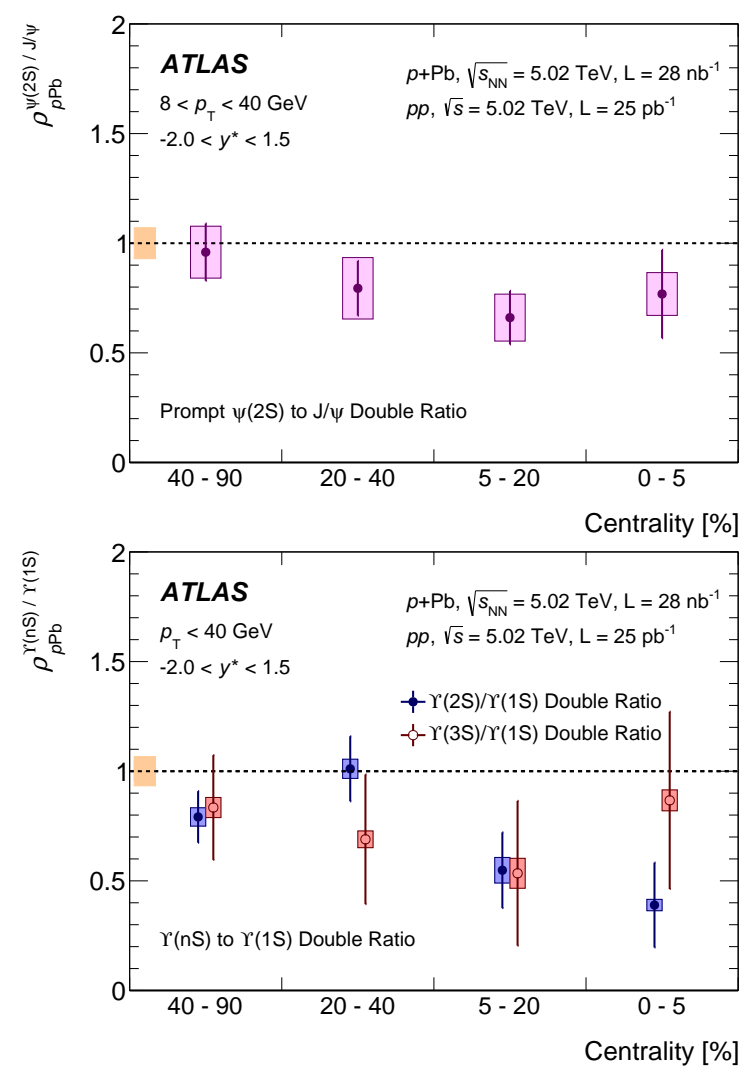

Figure 4: The double ratios $\rho_{p \mathrm{~Pb}}^{n S / 1 S}$ for prompt charmonia (top) and bottomonia (bottom) as a function of the collision centrality [7]. The vertical error bars and box sizes represent statistical and uncorrelated systematic uncertainties, respectively. The vertical size of the leftmost box corresponds to the total uncertainty of the $p p$ reference.
The kinematics of four particles in the final state can be described by the invariant mass of dimuon system $q^{2}$ and three helicity angles. The ATLAS analysis [9] followed the definition of angles $\theta_{K}, \theta_{L}$ and $\phi$ used by the LHCb collaboration in Ref. [10], for which the parameters of $B^{O}$ and $\bar{B}^{0}$ angular distributions are the same, hence it is possible to use a combined $B^{0}$ and $\bar{B}^{0}$ dataset.

The full angular differential decay rate can be written as a function of seven angular coefficients $S_{i}$ and the fraction of longitudinally polarised $K^{*}$ mesons, $F_{L}$. Because of a low number of signal candidates, transformations based on symmetry of trigonometric functions were used to simplify the angular distributions. The four resulting distributions depend only on $F_{L}, S_{3}$ and one of $S_{4}, S_{5}, S_{7}$ and $S_{8}$. A set of optimised parameters $P_{i}$ was also proposed to reduce the theoretical uncertainties that come from hadronic form factors and increase the sensitivity to new physics. The $P_{1}, P_{4}^{\prime}, P_{5}^{\prime}, P_{6}^{\prime}$ and $P_{8}^{\prime}$ can be computed from the measured values of $S_{i}$ and $F_{L}$.

The study used the $p p$ dataset recorded in 2012. Signal candidates were reconstructed from two charged tracks and two muons and a cut-based selection was applied to extract the sample for angular analysis. Requirements on vertex fit quality, lifetime significance $\tau / \sigma_{\tau}, B^{O}$ pointing angle and $p_{\mathrm{T}}$ of $K^{*}$ were used to suppress the combinatorial background.

Unbinned maximum-likelihood fit of the mass and angular distributions was performed. The shape of the signal mass PDF was modelled by a Gaussian with width scaled by the per-event mass error and the combinatorial background was described by exponential and Chebychev polynomial functions for mass and angular distributions, respectively. To increase the fit stability, a mass fit was done first and the extracted nuisance parameters (signal and background yields, parameters of the $B^{O}$ mass distribution) were fixed in the next step which added $\cos \theta_{K}, \cos \theta_{L}$ and $\phi$ in the likelihood.

The measurement was done in six bins of $q^{2}$ in $[0.04,6] \mathrm{GeV}^{2}$, where three bins overlapped. The total number of fitted signal candidates was $(342 \pm 39)$.

The largest systematic uncertainties came from partially reconstructed decays that enter the signal region and peak in the $\cos \theta_{K}$ and $\cos \theta_{L}$ distributions. Other contributions are from the parametrization of the signal acceptance, modelling of background angular distributions, tracking and ID alignment, non-resonant $B^{O} \rightarrow$ $K^{+} \pi^{-} \mu^{+} \mu^{-}$decay, intrinsic fit bias, nuisance parameters and assigning the wrong flavour to $B^{0}$ and $\bar{B}^{O}$.

A subset of results is shown in Figure 5. In general they agree with the predictions of the SM and the most significant deviations were observed for $P_{4}^{\prime}, P_{5}^{\prime}$ and $P_{8}^{\prime}$ in one $q^{2}$ bin each. The measured values of $P_{4}^{\prime}$ and $P_{5}^{\prime}$ 
for $q^{2} \in[4,6] \mathrm{GeV}^{2}$ are $2.7 \sigma$ away from the calculation by Descotes-Genon et al. [11] and a deviation of $1.9 \sigma$ from the same prediction is observed for $P_{8}^{\prime}$ for $q^{2} \in[2,4] \mathrm{GeV}^{2}$. The $P_{5}^{\prime}$ deviation is consistent with the one reported by the LHCb collaboration [10]. However, the presented results are statistically limited and measurements with a larger dataset collected during the LHC Run 2 are planned.

\section{Acknowledgements}

The author acknowledges support of grant LTT17018 from the Ministry of Education, Youth and Sports of the Czech Republic.

\section{References}

[1] ATLAS Collaboration, The ATLAS Experiment at the CERN Large Hadron Collider, JINST 3 (2008) S08003.

[2] D0 Collaboration, Evidence for a $B_{s}^{0} \pi^{ \pm}$state, Phys. Rev. Lett. 117 (2) (2016) 022003. arXiv:1602.07588.

[3] LHCb Collaboration, Search for Structure in the $B_{s}^{0} \pi^{ \pm}$Invariant Mass Spectrum, Phys. Rev. Lett. 117 (15) (2016) 152003. arXiv: 1608.00435 .

[4] CMS Collaboration, Search for the $\mathrm{X}(5568)$ state decaying into $\mathrm{B}_{\mathrm{s}}^{0} \pi^{ \pm}$in proton-proton collisions at $\sqrt{s}=8 \mathrm{TeV}$, Phys. Rev. Lett. 120 (20) (2018) 202005. arXiv:1712.06144.

[5] CDF Collaboration, A search for the exotic meson X(5568) with the Collider Detector at Fermilab, Phys. Rev. Lett. 120 (20) (2018) 202006. arXiv:1712.09620.

[6] ATLAS Collaboration, Search for a Structure in the $B_{s}^{0} \pi^{ \pm}$Invariant Mass Spectrum with the ATLAS Experiment, Phys. Rev. Lett. 120 (20) (2018) 202007. arXiv:1802.01840.

[7] ATLAS Collaboration, Measurement of quarkonium production in proton-lead and proton-proton collisions at $5.02 \mathrm{TeV}$ with the ATLAS detector, Eur. Phys. J. C78 (3) (2018) 171. arXiv:1709.03089.

[8] ALICE Collaboration, Rapidity and transverse-momentum dependence of the inclusive $\mathrm{J} / \psi$ nuclear modification factor in $\mathrm{p}-\mathrm{Pb}$ collisions at $\sqrt{s_{N N}}=5.02 \mathrm{TeV}$, JHEP 06 (2015) 055 . arXiv:1503.07179.

[9] ATLAS Collaboration, Angular analysis of $B_{d}^{0} \rightarrow K^{*} \mu^{+} \mu^{-}$decays in $p p$ collisions at $\sqrt{s}=8 \mathrm{TeV}$ with the ATLAS detector, arXiv:1805.04000.

[10] LHCb Collaboration, Angular analysis of the $B^{0} \rightarrow K^{* 0} \mu^{+} \mu^{-}$ decay using $3 \mathrm{fb}^{-1}$ of integrated luminosity, JHEP 02 (2016) 104. arXiv: 1512.04442 .

[11] S. Descotes-Genon, L. Hofer, J. Matias, J. Virto, On the impact of power corrections in the prediction of $B \rightarrow K^{*} \mu^{+} \mu^{-}$observables, JHEP 12 (2014) 125. arXiv:1407.8526.

[12] S. Jäger, J. Martin Camalich, Reassessing the discovery potential of the $B \rightarrow K^{*} \ell^{+} \ell^{-}$decays in the large-recoil region: SM challenges and BSM opportunities, Phys. Rev. D 93 (1) (2016) 014028. arXiv:1412.3183.

[13] M. Ciuchini, M. Fedele, E. Franco, S. Mishima, A. Paul, L. Silvestrini, M. Valli, $B \rightarrow K^{*} \ell^{+} \ell^{-}$decays at large recoil in the Standard Model: a theoretical reappraisal, JHEP 06 (2016) 116. arXiv: 1512.07157.

[14] H. S. Shao, H. Han, Y. Q. Ma, C. Meng, Y. J. Zhang, K. T. Chao, Yields and polarizations of prompt $J / \psi$ and $\psi(2 S)$ production in hadronic collisions, JHEP 05 (2015) 103. arXiv:1411.3300.
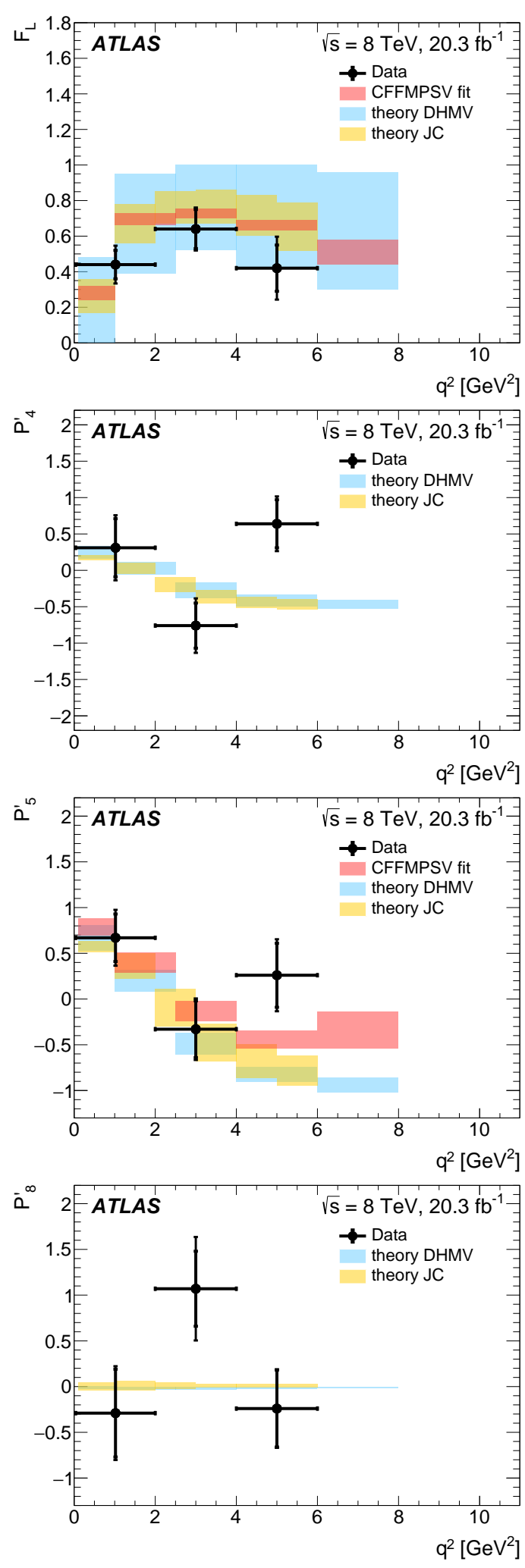

Figure 5: Measured values of $F_{L}, P_{4}^{\prime}, P_{5}^{\prime}$ and $P_{8}^{\prime}$ parameters in $B^{0} \rightarrow$ $K^{*} \mu^{+} \mu^{-}$decay [9]. They are compared with theoretical predictions of the SM: Descotes-Genon et al. (DHMV [11]) and Jäger and Martin Camalich (JC [12]) use the QCD factorisation with different treatment of long-distance effects, while Ciuchini et al. (CFFMPSV [13]) performed a compatibility check of the $\mathrm{LHCb}$ result [10] with theoretical predictions. The statistical and total uncertainties for data are shown by the inner and total error bar. 